\title{
LIBRI NOVI
}

J. M. Elliot, I977. Some methods for the statistical analysis of samples of benthic invertebrates. Freshw. Biol. Ass., Sc. Publ. no. 25. £ 2.00.

The book describes the methods of using mathematical frequency distributions (positive binomial, normal, Poisson, negative binomial) as models for samples from a population. Various indices are given by means of which one can decide whether a population has a regular, a random, or a contagious distribution. Attention is paid to the effect of sample size on the conclusion to be made. Sample quadrat sizes smaller than the clump sizes in a contagious distribution may give rise to the conception of a random or regular distribution. The methods to calculate the mean of the different types of distributions and its confidence limits are discussed in detail. In this new edition it is suggested to apply the factor to calculate the confidence limits of the derived mean of the contagious distribution to the arithmetic mean. This is the best choice, because otherwise the arithmetic mean may lie outside the confidence limits of the derived mean. In behalf of comparing two or more samples some parametric and non-parametric methods are given. Though common in the handbooks, they are brought now clearly in relation to population research. The last chapter deals with the planning of the sampling scheme and discusses how the diversity of the biotope can be taken into account by choosing between random, stratified, or systematic sampling.

In this edition of the book an index and an appendix with calculation diagrams have been added. The list of references has been extended with ten items. The book has a greater usability than the title suggests, because, for instance, the presented methods are usable for terrestrial organisms as well. Therefore this book is very valuable for all biologists studying patterns of distributions in populations of animals or plants.

A. G. Vlasblom 